\title{
Sidelobe Reduction of Unequally Spaced Arrays for 5G Applications
}

\author{
N. A. Zainal ${ }^{1,2}$, M. R. Kamarudin ${ }^{2}$, Y. Yamada ${ }^{3}$, Norhudah Seman ${ }^{2}$, Mohsen Khalily $^{4}$ and M. Jusoh ${ }^{5}$ \\ ${ }^{1}$ Department of Polytechnic, Ministry of Education Malaysia, Presint 4, 62100 W.P. Putrajaya, Malaysia. \\ Email: adam76784@gmail.com.my \\ ${ }^{2}$ Wireless Communication Center (WCC), Universiti Teknologi Malaysia (UTM), 81310 Skudai, Johor, Malaysia. \\ Email: ramlee@fke.utm.my \\ ${ }^{3}$ Malaysia-Japan International Institute of Technology (MJIIT), Universiti Teknologi Malaysia (UTM), 54100 K. L., Malaysia \\ Email: ndayamada@yahoo.co.jp \\ ${ }^{4}$ Institute for Communication Systems (ICS), home of the 5G Innovation Centre, Department of Electronic Engineering, \\ University of Surrey, Guildford GU2 7XH, U.K. \\ Email: m.khalily@surrey.ac.uk \\ ${ }^{5}$ School of Computer and Communication Engineering, Universiti Malaysia Perlis, Perlis. \\ Email: muzammil@unimap.edu.my
}

\begin{abstract}
Unequally spaced arrays technique offers an alternative for limited sidelobe level reduction compared to conventional array antennas with equally spaced elements. In this paper, the abilities and design of microstrip linear array antenna, fed by multiport feeding with uniform excitation coefficient in all array elements are presented for sidelobe level reduction at $28 \mathrm{GHz}$. By using the proximity coupled feed, simulation result gave $-10 \mathrm{~dB}$ impedance bandwidth of $1.42 \mathrm{GHz}$ and reflection coefficient of $\mathbf{- 3 5 . 5} \mathrm{dB}$ has been achieved. The sidelobe level at broadside decreased from $-11.77 \mathrm{~dB}$ to $-14.76 \mathrm{~dB}$ $(N=4)$ and $-12.77 \mathrm{~dB}$ to $-15.98 \mathrm{~dB}(N=8)$ with unequally spaced array. This feature is suitable for $5 \mathrm{G}$ applications.
\end{abstract}

Index Terms-5G antenna mobile, millimeter wave, unequally spaced array.

\section{INTRODUCTION}

With the evolution of the fifth generation $(5 \mathrm{G})$ mobile system, there will be a great deal of challenges which call for a higher capacity and a better performance in the $5 \mathrm{G}$ environment. Operating in a new frequency band in millimeter wave communication system is one of the ways to get rid of the overcrowded lower frequency range. This frequency band provides the necessary bandwidth [1] $(3 \mathrm{GHz} \sim 300 \mathrm{GHz})$ [2] for high speed and efficient use. Previous research has proposed the use of $28 \mathrm{GHz}$ band, which is currently used for mobile applications [3]. In 5G, the short wavelengths also introduce significant deployment challenges. In order to increase the antenna performance and control the radiation pattern, a number of elements, such as the spacing between elements and excitation coefficients (amplitude and phase) as well as antenna elements are important. Apart of producing narrow beam antenna low sidelobe level is also needed to reduce the interference radiation in mobile system. Therefore, the use of unequally spaced arrays [4] is promising by tapering the amplitude of the element of excitation.

Equally spaced array antenna has been widely proposed in the scientific literature. Schuman and Strait [5] presented a design of unequally spaced arrays with nearly equal sidelobe using computerized design technique. The spacing and amplitudes of the array elements are allowed to vary in order to achieve a specified sidelobe level. Sandrin and Hague [6] have suggested the method to maximize amplitude of the excitation element in linear array when specified sidelobe and/or beamwidth constraints are required by the use of nonuniform element spacings. However, for King, Packard and Thomas [7], the method was used to reduce the grating lobes and the results of using unequal spacing in a linear array are promising. In this paper, numerical data of reduction of sidelobe level using unequally spaced arrays to avoid a complicated feed network is presented. The microstrip array antenna fed by multiport structure for multi element antenna is designed with uniform excitation coefficients.

\section{Structure Of Patch ANTENNA DESIGN}

Microstrip patch antenna, which is known inherently as a narrow bandwidth and may introduce spurious radiation especially at a high frequency. In order to enhance the bandwidth, proximity coupled [8] feed technique was used. This is to avoid the physical contact between the patch and the feeding network so that spurious radiation can be eliminated and a wider bandwidth can be achieved to fulfil the $5 \mathrm{G}$ requirements.

\section{A. Single element antenna design}

The single microstrip patch antenna structure is shown in Fig. 1. The proximity coupled feed was implemented with a patch element on $0.254 \mathrm{~mm}$ superstrate and feed line on 0.254 $\mathrm{mm}$ substrate. The patch element with a dimension of $W=3.27$ $\mathrm{mm}$ and $L=3.27 \mathrm{~mm}$ was designed to operate at $f_{0}=28 \mathrm{GHz}$. The printed circuit board of Rogers RT/duroid $5880\left(\varepsilon_{r l}=\varepsilon_{r 2}\right.$ $=2.2, h_{1}=h_{2}=0.254 \mathrm{~mm}$ and $\left.\tan \delta=0.0009\right)$ with a dimension of $15 \mathrm{~mm} \times 15 \mathrm{~mm} \times 0.254 \mathrm{~mm}^{3}$ (Length $\mathrm{x}$ Width $\mathrm{x}$ Height) was used. 


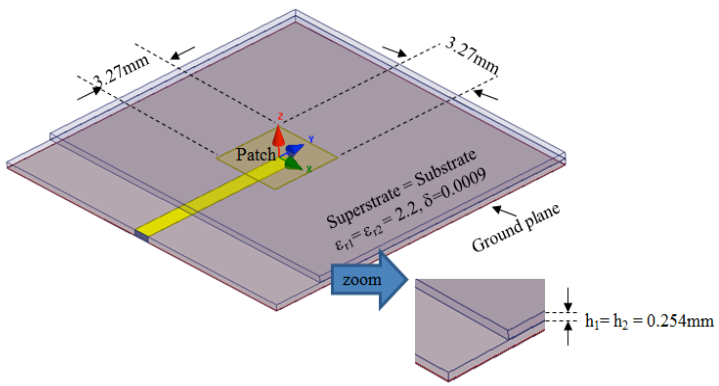

Fig. 1. Structure for single patch antenna at $28 \mathrm{GHz}$

1) Simulation result: Fig. 2(a) shows a cross sectional view of $3 \mathrm{D}$ radiation pattern with a gain of $7.4 \mathrm{dBi}$. The proposed design offers a broadside radiation pattern with a simple multiport feed structure. The radiation pattern is very broad in single element, as shown in Fig. 2(b). The H-plane and E-plane radiation patterns were obtained by setting $\phi=0^{0}$ and $\theta=90^{\circ}$. However, enlarging the size of the element will increase the gain of antenna and narrow down the beam pattern. Based on the previous research, at $28 \mathrm{GHz},-10 \mathrm{~dB}$ impedance bandwidth of $<1 \mathrm{GHz}$ is achieved using microstrip patch antenna with inset feedline. However, with proximity coupled feed, a bandwidth of $1.42 \mathrm{GHz}$ is achieved with a reflection coefficient of $-35.5 \mathrm{~dB}$, as shown in Fig. 2(c).

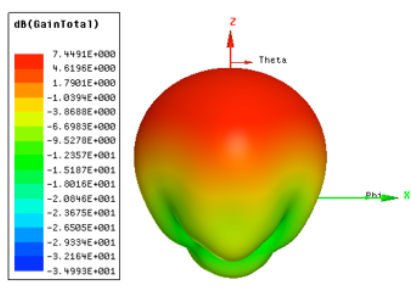

(a)

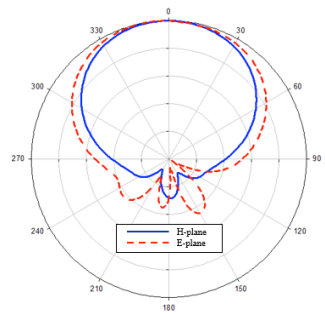

(b)

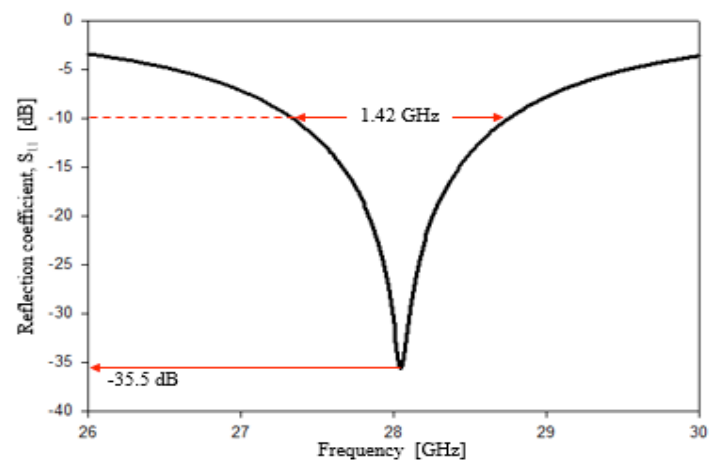

(c)

Fig. 2. Simulated structure of single patch antenna at $28 \mathrm{GHz}$ (a) 3D pattern (b) Radiation pattern (c) Reflection coefficient $\left(\mathrm{S}_{11}\right)$

\section{B. Array configurations}

A linear array of $N$ identical elements with a uniform spacing $d$, positioned along the $\mathrm{x}$-axis of a conventional coordinate system is shown in Fig. 3. The microstrip linear array antenna, which was fed by multi-port, consists of four elements, and has uniform amplitude and phase in all array elements. In this case, feed network has an equal feed line and length. Therefore, there was no frequency dependence because of the uniform excitation coefficient (amplitude and phase). The equally spaced array (ESA) was used as the reference. Two array configurations of unequally-spaced array, which consist of unequally spaced array 1 (USA1) and unequally spaced array 2 (USA2) have been chosen for examination. The array factor of linear array is given by (1) [9].

$$
A F=A_{0} \sum_{n=0}^{N-1} e^{j n \psi}=A_{0}\left(1+e^{j \psi}+\cdots+e^{j(N-1) \psi}\right)
$$

where $\psi=k d \cos \theta+\delta, k=2 \pi / \lambda$ is the free space wave number, $d$ is element spacing and $N$ is the number of elements. According to broadside array, the phase shift of $\delta$ is equal to zero $(\delta=0)$.

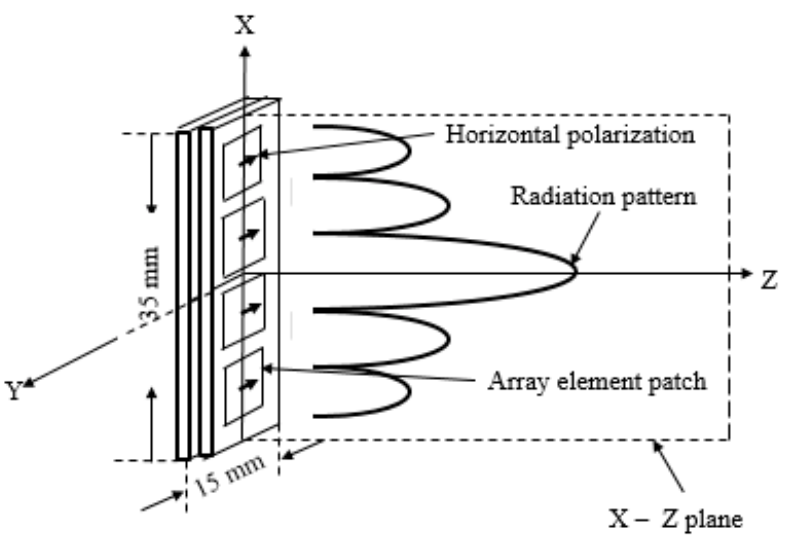

(a)

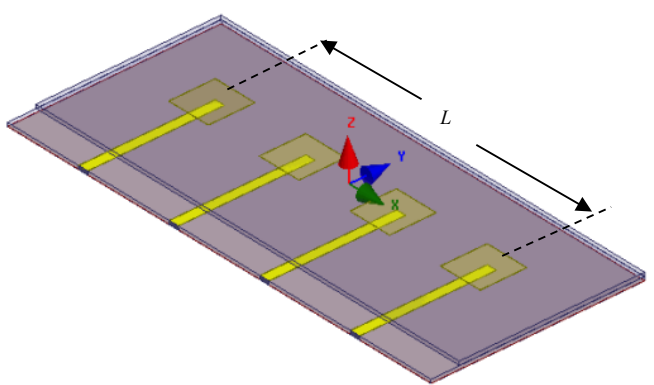

(b)

Fig. 3. Array configuration (a) Calculated array configuration (b) 3D view 
1) Equally spaced array (ESA): Most antenna arrays employ equal spacing between elements. However, since the purpose of this paper is to investigate unequally spaced array, the radiation pattern of uniform spaced arrays can be used as the reference pattern. The equally element spacing is set with $d=0.7 \lambda_{0}\left(\lambda_{0}\right.$; wavelength at $\left.f_{0}\right)$, as shown in Fig. 4 . However, a better performance of sidelobe level of unequally spaced array is expected.

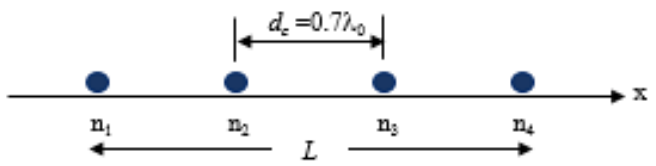

Fig. 4. Array antenna spacing configuration for Equally spaced array (ESA)

2) Unequally spaced array: In unequally spaced arrays, the element spacing at the center element $\left(d_{c}\right)$ was $0.6 \lambda_{0}$ for unequally spaced array 1 (USA1) while for unequally spaced array 2 (USA 2), it was $0.5 \lambda_{0}$. The total array length $(L)$ is constant as $L=3 \times 0.7 \lambda_{0}(=22.47 \mathrm{~mm})$, the value was unchanged for $N=4$. Array spacings are considered symmetrical around the array center. For the case of unequally spaced array antenna for $N=4$, element spacing is given in (2).

$$
d_{i}=d_{c}+i . \Delta d(i=\text { number of element }=0 \sim 1)
$$

$\Delta d$ is determined with the following relation.

$$
L=3 d_{c}+2 \Delta d
$$

When $d_{c}$ is given, $\Delta d$ is determined by (3). The different arrangements of unequally array element spacing are shown in Fig. 5 in which the radiation pattern, designed in the vertical plane, is shown in $\mathrm{x}-\mathrm{z}$ plane.

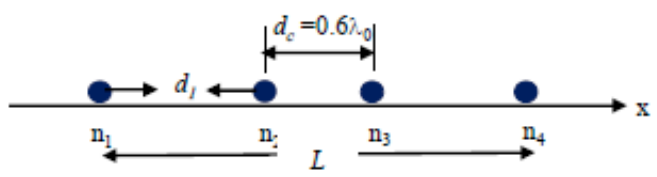

(a)

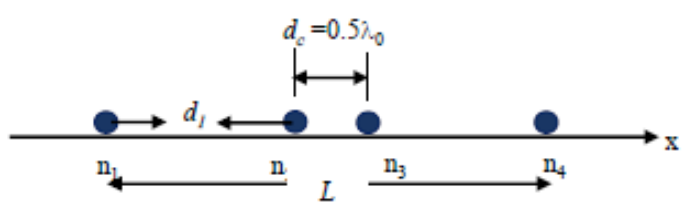

(b)

Fig. 5. Array antenna spacing configuration (a) Unequally spaced array 1 (USA1) (b) Unequally spaced array 2 (USA2)

\section{SIDELOBE CHARACTERISTICS}

Based on the three different array antenna spacing configurations, the performance of the antenna will be discussed in the following paragraph.

\section{A. Sidelobe performance}

With calculation, a High Frequency Structure Simulator (HFSS) was employed. The radiation pattern in the $\mathrm{x}-\mathrm{z}$ plane was emphasized. Fig. 6 shows the $2 \mathrm{D}$ radiation pattern with different center spacing of antenna. A sidelobe level reduction was observed when there was a higher amplitude current distribution at the end of the array. The sidelobe level for ESA was $-11.77 \mathrm{~dB}$ and it has reduced to $-13.50 \mathrm{~dB}$ and $-14.76 \mathrm{~dB}$ for USA1 and USA2 respectively. However, the sidelobe level for the sidelobe that was farther from the main lobe has increased considerably. At the same time, the gain has decreased slightly while there was not much difference in HPBW for the three array configurations. Fig. 7 shows the comparison of $2 \mathrm{D}$ radiation pattern for four and eight elements. The total array length $(L)$ for $N=8$ was the same as $L=7 \times 0.7$ $\lambda_{0}(=52.43 \mathrm{~mm})$. Based on the result, the sidelobe level and beamwidth were found to decrease when the number of element increases and there was a reduction in sidelobe level when unequally spaced inter element was applied. In addition, the calculation of simulated result shows that the antenna has a radiation efficiency of $-0.031 \mathrm{~dB}$. Therefore, the proposed antenna has an efficiency of $0.99 \%$. Table 1 has summarized the simulated results for sidelobe performance.

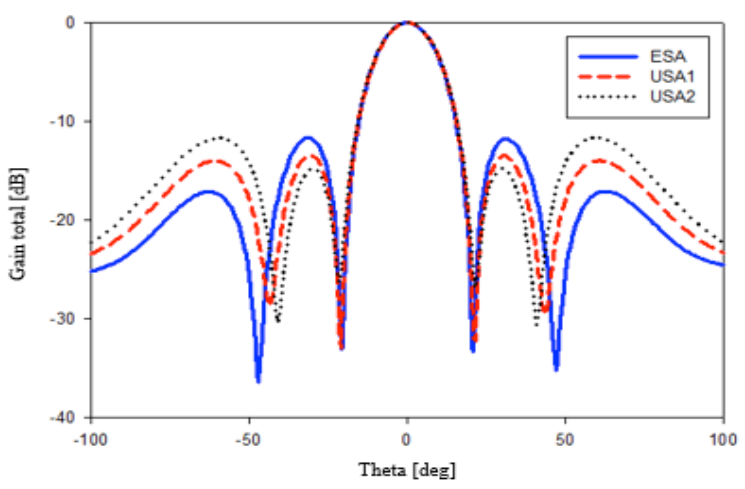

Fig. 6. Sidelobe characteristics for four elements

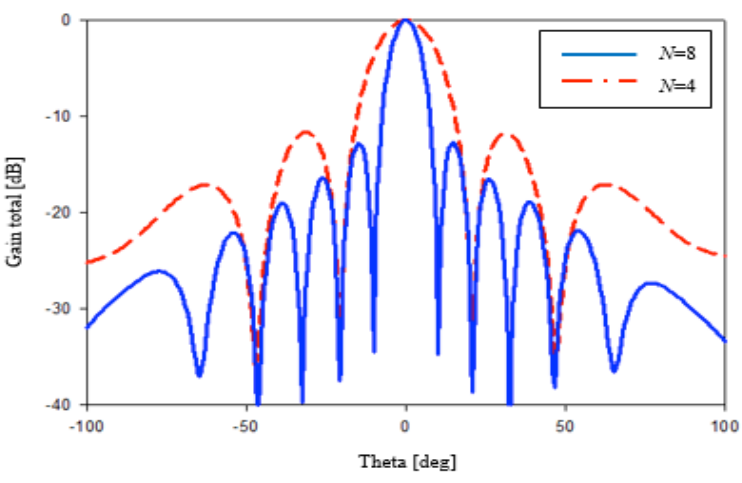

(a) 


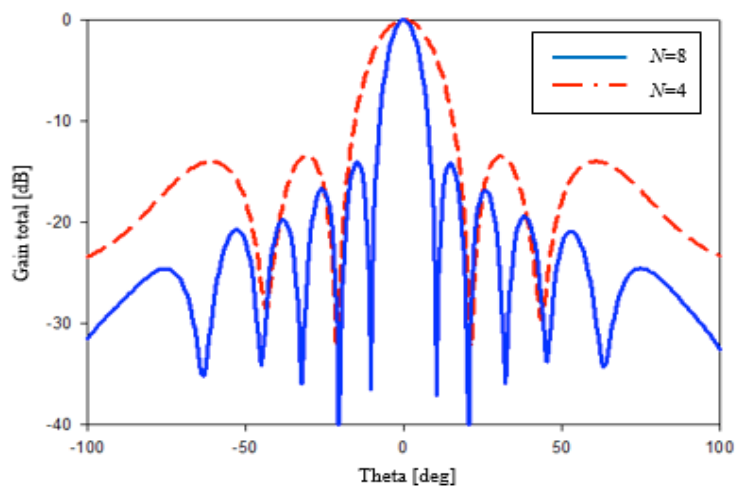

(b)

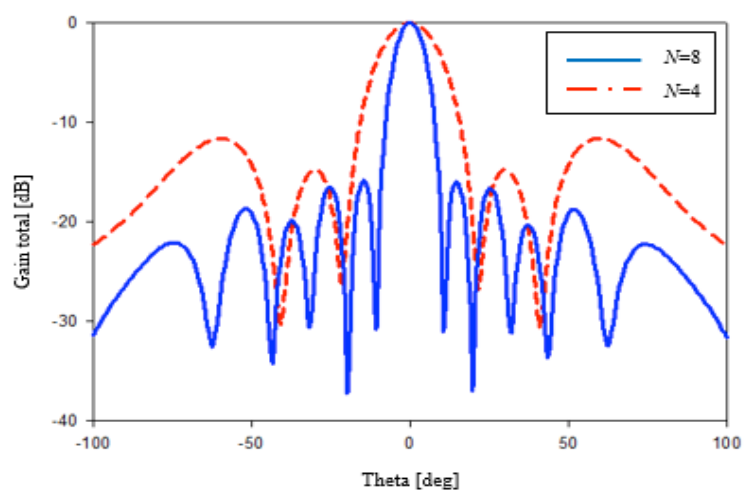

(c)

Fig. 7. Comparison of sidelobe characteristics (a) Equally spaced array (ESA) (b) Unequally spaced array 1 (USA1) (c) Unequally spaced array 2 (USA2)

TABLE I. SUMMARIZED SIMULATED RESULTS

\begin{tabular}{|c|c|c|c|c|c|c|}
\hline \multirow{3}{*}{$\begin{array}{c}\text { Antenna } \\
\text { parameter }\end{array}$} & \multicolumn{6}{|c|}{ Array configuration } \\
\hline & \multicolumn{2}{|c|}{$\begin{array}{c}E S A \\
\left(d_{c}=0.7 \lambda_{0}\right)\end{array}$} & \multicolumn{2}{|c|}{$\begin{array}{c}\text { USA1 } \\
\left(d_{c}=0.6 \lambda_{0}\right)\end{array}$} & \multicolumn{2}{|c|}{$\begin{array}{c}\text { USA2 } \\
\left(d_{c}=0.5 \lambda_{0}\right)\end{array}$} \\
\hline & $N=4$ & $N=8$ & $N=4$ & $N=8$ & $N=4$ & $N=8$ \\
\hline $\begin{array}{l}\text { Sidelobe } \\
\text { level (dB) }\end{array}$ & -11.77 & -12.77 & -13.50 & -14.18 & -14.76 & -15.98 \\
\hline $\operatorname{HPBW}\left({ }^{0}\right)$ & 18.43 & 9.01 & 18.51 & 9.12 & 18.70 & 9.22 \\
\hline Gain $(\mathrm{dBi})$ & 12.49 & 15.47 & 12.49 & 15.46 & 12.43 & 15.43 \\
\hline
\end{tabular}

\section{CONCLUSION}

In this study, the effects of unequally spacing, as reflected by the sidelobe levels for linear array antenna, have been investigated. The simulations were carried out using High Frequency Structure Simulator (HFSS) version 16.0. Based on the results, it can be concluded that the sidelobe level can be reduced rather well with the change of the spacing between elements. Using proximity coupled feed will also enable a higher bandwidth application, with a wider bandwidth of more than $1 \mathrm{GHz}$. For mobile applications, $28 \mathrm{GHz}$ is considered the requirement for $5 \mathrm{G}$ antennas. The gain and degree of HPBW can be improved by enlarging an array size so as to provide a higher gain and a more directive beam pattern. Based on the simulated results presented in this paper, it was obvious that a considerable reduction of sidelobe level is possible with the change of the distance between elements. The fact that there was no frequency dependence of feeding network for the multiport feeding techniques used in this work has opened up a new avenue for the authors' further research, focusing on multi band mobile base station antennas for $5 \mathrm{G}$ mobile applications.

\section{ACKNOWLEDGMENT}

The authors would like to thank the Ministry of Education and Universiti Teknologi Malaysia for sponsoring this work under FRGS Grant (Vot 4F283) and RUG (00M69\&11H59) and also the scholarship grant for PhD study at UTM.

\section{REFERENCES}

[1] R.B. Waterhouse, D. Novak, A. Nirmalathas, and C. Lim, "Broadband printed sectorized coverage antennas for millimeter-wave wireless applications," IEEE Transactions on Antennas and Propagation, vol. 50 (1), pp. 12-16, January 2002.

[2] Cheng-Xiang Wang, F. Haider, Xiqi Gao, Xiao-Hu You, Yang Yang, Dongfeng Yuan, H. Aggoune, H. Haas, S. Fletcher, and E. Hepsaydir, "Cellular architecture and key technologies for 5G wireless communication networks," IEEE Communication Magazine, vol. 52(2), 2014, pp. 122-130.

[3] T.S. Rappaport, Shu Sun, K. Mayzus, Hang Zhao, Y. Azar, K. Wang, G.N Wong, J.K Schulz, M. Samimi, and F. Gutierrez, "Millimeter wave mobile communications for $5 \mathrm{G}$ cellular: It Will Work!," IEEE Access, vol. 1, 2013, pp. 335-349.

[4] R.E. Collin, F.J. Zucker, Antenna theory: part 1, Mc Graw-Hill, 1969.

[5] H.K. Schuman and B.J. Strait, "On the design of unequally spaced arrays with nearly equal sidelobes," IEEE Transactions Antennas and Propagation (Communications), vol. 16(4), July 1968, pp. 493-494.

[6] W. Sandrin and D. Hague, "Design of arrays with unequal spacing and partially uniform amplitude taper", IEEE Transactions Antennas and Propagation, vol. 17(5), 1969, pp. 642-644.

[7] D.D. King, R.F. Packard, and R.K. Thomas, "Unequally spaced, broadband antenna arrays", IRE Transactions of Antennas and Propagation, vol. 8(4), 1960, pp. 380-384.

[8] D.M. Pozar and B. Kaufman, "Increasing the bandwidth of a microstrip antenna by proximity coupling”, Electronics Letter, vol. 23(8), 1987, pp.368-369.

[9] Warren L. Stutzman and Gary A. Thiele, Antenna theory and design, 3rd ed., Wiley, 2013, pp.279. 